\title{
Association, effects and validation of polymorphisms within the NCAPG - LCORL locus located on BTA6 with feed intake, gain, meat and carcass traits in beef cattle
}

\author{
Amanda K Lindholm-Perry ${ }^{1 *}$, Andrea K Sexten ${ }^{1,2}$, Larry A Kuehn ${ }^{1}$, Timothy PL Smith ${ }^{1}$, D Andy King ${ }^{1}$, \\ Steven D Shackelford ${ }^{1}$, Tommy L Wheeler ${ }^{1}$, Calvin L Ferrell ${ }^{1,3}$, Thomas G Jenkins ${ }^{1,3}$, Warren M Snelling ${ }^{1}$ and \\ Harvey C Freetly ${ }^{1}$
}

\begin{abstract}
Background: In a previously reported genome-wide association study based on a high-density bovine SNP genotyping array, 8 SNP were nominally associated $(P \leq 0.003)$ with average daily gain $(A D G)$ and 3 of these were also associated $(P \leq 0.002)$ with average daily feed intake (ADFI) in a population of crossbred beef cattle. The SNP were clustered in a $570 \mathrm{~kb}$ region around $38 \mathrm{Mb}$ on the draft sequence of bovine chromosome 6 (BTA6), an interval containing several positional and functional candidate genes including the bovine $\angle A P 3, N C A P G$, and LCORL genes. The goal of the present study was to develop and examine additional markers in this region to optimize the ability to distinguish favorable alleles, with potential to identify functional variation.

Results: Animals from the original study were genotyped for 47 SNP within or near the gene boundaries of the three candidate genes. Sixteen markers in the NCAPG-LCORL locus displayed significant association with both ADFI and ADG even after stringent correction for multiple testing $(P \leq 005)$. These markers were evaluated for their effects on meat and carcass traits. The alleles associated with higher ADFI and ADG were also associated with higher hot carcass weight (HCW) and ribeye area (REA), and lower adjusted fat thickness (AFT). A reduced set of markers was genotyped on a separate, crossbred population including genetic contributions from 14 beef cattle breeds. Two of the markers located within the LCORL gene locus remained significant for ADG $(P \leq 0.04)$.

Conclusions: Several markers within the NCAPG-LCORL locus were significantly associated with feed intake and body weight gain phenotypes. These markers were also associated with HCW, REA and AFT suggesting that they are involved with lean growth and reduced fat deposition. Additionally, the two markers significant for ADG in the validation population of animals may be more robust for the prediction of ADG and possibly the correlated trait ADFl, across multiple breeds and populations of cattle.
\end{abstract}

\section{Background}

Feed intake and average daily gain are economically important phenotypes in beef cattle. The ability to genetically select for animals that better suit a producer's needs in terms of the amount of feed an animal consumes and the gain that can be expected would

\footnotetext{
* Correspondence: Amanda.Lindholm@ars.usda.gov

'United States Department of Agriculture, Agricultural Research Service, U.S. Meat Animal Research Center, State Spur 18D, Clay Center, NE 68933, USA Full list of author information is available at the end of the article
}

benefit the industry. However, it is critical that markers used to select for feed and growth traits do not adversely affect other traits including meat quality, carcass traits or reproduction.

Quantitative trait loci (QTL) for cattle growth traits such as birth weight, body length, carcass weight, and longissimus muscle area (LMA) have been detected on bovine chromosome 6 in several different populations of animals. Nkrumah et al. [1] discovered a QTL at $42 \mathrm{cM}$ for average daily gain (ADG) in a composite population

\section{Biomed Central}


of steers. Body length at birth and birth weight QTL were identified by Gutiérrez-Gil et al. [2] at 38 and 39 $\mathrm{Mb}$, respectively, in a Charolais $\times$ Holstein crossbred population. Several other QTL have been detected for birth weight in this same region [3-6]. In addition to live animal growth, length and weight QTL, others have found that QTL for carcass and meat quality traits are located in this region. Takasuga et al. [7] detected a QTL for carcass weight at $38 \mathrm{cM}$, Setogutchi et al. [8] found QTL for carcass weight and LMA at $39.1 \mathrm{cM}$ and McClure et al. [6] identified a QTL for ribeye area (REA) at $41 \mathrm{cM}$. There is clear evidence that the region on BTA6 between 30 and $42 \mathrm{cM}$ is involved in growth and size phenotypes across multiple breeds of cattle.

We have also detected a region on chromosome 6 that is correlated with feed intake and gain located within $37.96-38.53 \mathrm{Mb}$ in a crossbred population of cattle [9]. There are seven genes on the Btau 4.0 genome assembly in this region, including leucine aminopeptidase 3 (LAP3), mediator complex subunit 28 (MED28), LOC523874, C6H4Orf30, non-SMC condensin I complex, subunit G (NCAPG), and LOC540095 (bovine ortholog of ligand dependent nuclear receptor corepressor-like, $L C O R L)$ ) $L A P 3$ is an aminopeptidase that catalyzes the removal of amino acids from intracellular proteins and peptides. LAP3 is most highly expressed in the bovine pineal gland, kidney, skin, intestine, mammary, and adipose tissues (UGID: 1959690, UniGene Bt.56962). MED28 is a subunit of the Mediator complex that interacts with RNA polymerase II and can interact with co-activators and co-repressors [10]. MED28 is most highly expressed in the bovine pineal gland, lyphoreticular tissue, uterus, abomassum, and expressed in lower quantities in the liver, reticulum, intestine and kidney (UGID:1230902, UniGene: Bt.29898). LOC523874 is homologous to the human family with sequence similarity 184, member B (FAM184B) for which there is little information. Recent SNP association studies suggest that NCAPG has a function in cattle growth $([8,11]$ possibly through a role in cell proliferation [12]. NCAPG is expressed in bovine pancreas, skin, intestine, liver, kidney, and ovary (UGID:3093465, UniGene Bt.100379). LCORL has recently been associated with human skeletal size [13] and height growth in infancy [14]. LCORL is also expressed in the liver, intestine in the bovine fetus and calf, and in the extraembryonic tissue of the bovine fetus (UGID:1484658, UniGene Bt.38533). Based on this information, we chose to evaluate $L A P 3, N C A P G$ and $L C O R L$ as potential positional candidate genes for cattle feed intake and growth.

The purpose of this study was to further refine the region on chromosome 6 that was previously identified by a SNP association as significant for feed intake and average daily gain in our population of steers [9]. While genetic markers predictive for feed intake and growth may be useful for the identification and selection of animals that are more efficient, attention must be given to the impact of these SNP on other production traits. Accordingly, we evaluated the markers that were highly significant for feed intake and gain for effects on carcass and meat quality traits. Here, we present a number of markers that may be useful for the genetic selection of animals of multiple breeds for feed and gain traits and illustrate that these markers appear to play a role in lean growth and fat deposition.

\section{Methods}

\section{Animals}

All animal procedures were reviewed and approved by the U.S. Meat Animal Research Center (USMARC) Animal Care and Use Committee. Procedures for handling cattle complied with those specified in the Guide for the Care and Use of Agricultural Animals in Agricultural Research and Teaching [15].

\section{Discovery population}

The breeding scheme used for the SNP discovery population of animals with feed efficiency phenotypes is described in Snelling et al. [9]. Briefly, purebred Angus (AN), Hereford (HH), Simmental (SM), Limousin (LM), Charolais (CH), Gelbvieh (GV) and Red Angus (AR) sires were mated by artificial insemination to composite MARC III (1/4 AN, 1/4 HH, 1/4 Pinzgauer, 1/4 Red Poll), $\mathrm{AN}$ and $\mathrm{HH}$ cows to produce progeny designated as $F_{1}$, born in 1999, 2000, and 2001. Female $F_{1}$ and the 2001-born $F_{1}$ males (AN, HH dams only) were kept for breeding, and mated in multiple-sire pastures to produce 2-, 3-and 4-breed cross progeny designated $\mathrm{F}_{1}{ }^{2}$. The $\mathrm{F}_{1}{ }^{2}$ calves were born in March through May of 2003 to 2007, from 3-year-old and older dams. Male calves were castrated within $24 \mathrm{~h}$ after birth. Calves were weaned in September at approximately $165 \mathrm{~d}$ of age [9]. Animals were screened and excluded for medical or health issues that may have affected either feed intake or gain phenotypes.

\section{SNP identification population}

Animals $(\mathrm{n}=24)$ used for resequencing of candidate genes $L A P 3, N C A P G$, and $L C O R L$ were selected from the discovery population of animals. These animals represented extreme feed intake and gain phenotypes.

\section{Validation population}

Validation animals $(\mathrm{n}=406)$ were derived from progeny in the USMARC continuous Germplasm Evaluation Program [16]. These progeny were part of a breeding program to develop purebred cattle of each of 14 breeds. In addition to the seven mentioned previously, these 
include Braunvieh, Shorthorn, Brahman, Maine Anjou, Santa Gertrudis, Salers, and Chi-Angus. Sires of each of these 14 breeds were mated to female descendants of cattle in the discovery population. This population did not include full or partial sibs from the discovery population. Approximately $60 \%$ of the validation animals were half-bloods sired by these seven new breeds and $40 \%$ were half- or three-quarter-bloods from the original seven breeds. Progeny were produced in three breeding seasons (Fall 2007, Spring 2008, and Fall 2008). Animals $(\mathrm{n}=29)$ were removed from the analysis due to chronic diagnoses of pneumonia, foot-rot, and bloat).

\section{Feed efficiency phenotypes}

Discovery population calves were familiarized to the test ration as described in Snelling et al. [9]. Briefly, beginning at $276 \pm 15 \mathrm{~d}$ of age, individual feed intake measurements were acquired with Calan Broadbent Feeding Systems (American-Calan-Broadbent, Northwood, NH). Animals were provided feed at $0800 \mathrm{~h}$ in the morning and were given ad libitum access. The mixed ration on a dry matter basis was $82.0 \%$ dry rolled corn, $10.6 \%$ ground alfalfa hay, $5.66 \%$ Soybean meal, $1.25 \%$ limestone, $0.40 \%$ urea, $0.06 \%$ salt, $0.007 \%$ trace minerals, $0.008 \%$ Vitamins A, D, and E, and 0.015\% Rumensin 80. Feed refusals were obtained each week.

Feed efficiency phenotypes for $\mathrm{F}_{1}{ }^{2}$ steers (discovery population) are described in detail in Snelling et al. [9]. Briefly, dry matter intake (DMI) was equal to cumulative dry matter intake for the 140-d feeding period. Average daily feed intake (ADFI) is the average daily dry matter intake (DMI/140). Individual animal quadratic regressions were fitted for body weight on time, and gain was calculated as the difference of BW predicted at $140 \mathrm{~d}$ and the intercept [17].

The steers used for the validation study were fed for either 84 or $148 \mathrm{~d}$. Feed intake for those fed for $84 \mathrm{~d}$ was measured using an Insentec system (Marknesse, The Netherlands). Diets were similar to those used in the discovery population. Dry matter intake was equal to the total DMI consumed for either the 84- or 148-d period and averaged to a daily basis. A linear regression was used to predict BW in steers fed for $84 \mathrm{~d}$ and a quadratic equation was used to predict BW in steers fed for $148 \mathrm{~d}$. Gain for both groups was calculated for the respective feeding periods as described in the discovery population above.

\section{Meat quality and carcass phenotypes (discovery population)}

$\mathrm{F}_{1}^{2}$ steers were serially harvested at a commercial processing plant as described by King et al. [18]. USDA yield and quality grade data were obtained by trained USMARC personnel after $36 \mathrm{~h}$ in the cooler at $0^{\circ} \mathrm{C}$.
Wholesale ribs were obtained as described by King et al. [18]. A posterior section of the ribeye was frozen at 14 $\mathrm{d}$ postmortem and a $2.54 \mathrm{~cm}$ thick steak was cut from the $11^{\text {th }}$ rib region. For slice shear force (SSF), steaks were thawed at $5^{\circ} \mathrm{C}$ for $24 \mathrm{~h}$ then cooked on a conveyorized electric belt grill to a final internal temperature of $71^{\circ} \mathrm{C}$ as described by Wheeler et al. [19]. SSF was determined as described by Shackelford et al. [20].

\section{Identification of SNP markers in LAP3, NCAPG and LCORL (SNP identification population)}

DNA was isolated from whole blood or buffy coats collected from steers selected from the discovery population of animals. Animals $(n=24)$ with extreme ADG or ADFI phenotypes were chosen for SNP discovery. Twelve animals with low ADG or ADFI values and twelve animals with high ADG or ADFI values were used. All seven breeds were represented in the animals with high and low phenotypes. None of the breeds were over-represented in the high and low groups. Angus and Hereford breeds each represented $\sim 30 \%$ of the breed composition in both groups. Simmental represented $12.5 \%$ in the low group and $16 \%$ in the high. Composition of the other breeds (Limousin, Gelbvieh, Red Angus, Charolais, MARCIII) ranged from 3-8\%.

Primer pairs for amplification of regions of $L A P 3$, NCAPG and LCORL from genomic DNA were designed using Primer 3 [21] (code available at http://frodo.wi. mit.edu). Cattle sequences used as templates for primer design were obtained from the chromosome 6 Btau 4.0 assembly. Oligonucleotide primers were synthesized by IDT (Integrated DNA Technologies, Coralville, IA). PCR was performed in a DNA engine Dyad ${ }^{\circledR}$ peltier thermal cycler (Bio-Rad, Hercules, CA). PCR reactions included 0.25 U Hot Star Taq polymerase (Qiagen, Valencia, CA); $1 \times$ supplied buffer; $1.5 \mathrm{mM} \mathrm{MgCl}_{2} ; 80 \mu \mathrm{M}$ dNTPs; $0.33 \mu \mathrm{M}$ each primer; and $25 \mathrm{ng}$ genomic DNA in $12 \mu \mathrm{l}$ reactions. Three microliters of the PCR reaction were electrophoresed in $2 \%$ agarose gels to determine quality of amplification, and the remainder was prepared for sequencing after treatment with $0.1 \mathrm{U}$ exonuclease I (USB, Cleveland, OH). Sequencing reactions were precipitated with $70 \%$ isopropanol and sequenced with an ABI 3730 capillary sequencer (Applied Biosystems, Foster City, CA). Bases were called with Phred and assembled into contigs with Phrap. Polymorphisms were identified using Polyphred and assessed using Consed http://www.phrap.org.

\section{Genotyping analysis}

Multiplex assays for the Sequenom MASSARRAY ${ }^{\circledR}$ instrument were designed with the MASSARRAY ${ }^{\circledR}$ Assay Design 3.0 software (Sequenom, San Diego, CA). Assays were designed for 47 SNPs in and around the 
LAP3, NCAPG, and LOC540095 (LCORL) gene loci. Each amplification primer contained a 10-base tag to yield amplification products with different masses. Amplicon lengths were between 90 and 120 bp. Reaction conditions were performed as recommended by Sequenom. A total of 1,137 animals with dry matter intake and average daily gain phenotypes and their sires were genotyped and used in the analyses after exclusion.

\section{Statistical analysis}

The data were analyzed using an animal model (MTDFREML) [22] that included the fixed effects of year and barn. Covariates of age and heterosis were also included. Calf and dam breed compositions were modeled with covariates for proportions AN, HH, SM, LI, $\mathrm{CH}, \mathrm{GV}, \mathrm{AR}$, and MARC III. Covariates for expected calf heterosis were computed from parental breed composition. The number of copies of the minor allele was fitted separately in the association model. Polygenic and breed effects were included to reduce the effects of family structure on breed- and family-specific alleles $[23,24]$. Variance components for polygenic effects and error were estimated using MTDFREML [22]. Nominal significance values for each SNP were computed. A Bonferroni correction for multiple testing was applied to the $P$-values by multiplying the $P$-value by the number of markers tested $(n=47)$. Analyses of significant SNP in the validation population followed the same model definition.

\section{Linkage disequilibrium (LD) analysis}

Linkage disequilibrium $\left(\mathrm{r}^{2}\right)$ was defined for the 47 SNP on chromosome 6 using Haploview 4.0 software [25] http://www.broad.mit.edu/mpg/haploview/index.php. Blocks of LD were based on pairwise LD values. Haploview settings were as follows: the exclusion of animals with $>50 \%$ missing genotypes, ignoring pairwise comparisons of markers $>500 \mathrm{~kb}$ apart, the percentage of genotypes $\geq 50 \%$ and minimum minor allele frequency of 0.001 .

\section{Results}

\section{Candidate gene SNP identification}

The genes LAP3, NCAPG and LCORL were chosen as positional and functional candidate genes in this region. The coding regions and some intronic regions of these genes were sequenced and polymorphisms are shown in Table 1. A polymorphism in the NCAPG gene identified previously by Setoguchi et al. [8] (p.I442M) was also detected in our population of animals (marker 83714_224 in this study). In addition to this polymorphism, we identified a second non-synonymous alteration in NCAPG (p.L877M). A 3 base pair deletion resulting in an in-frame deletion of a glutamic acid (p.E47del) and a non-synonymous alteration (p.D92E) were detected in LAP3 (Table 1).

\section{Discovery population analyses}

A total of 21 out of the 39 SNP identified within the LAP3 $(\mathrm{n}=9), N C A P G(\mathrm{n}=8)$ and LCORL $(\mathrm{n}=4)$ genes were multiplexed into Sequenom genotyping assays. In addition to these, another 26 publicly available SNP located up- and down-stream of these genes that were not used on the BovineSNP50 BeadChip [26-28], were also placed into the genotyping assays. The population of steers genotyped previously [9] with the BovineSNP50 BeadChip $(n=1,066)$ and their sires $(n=71)$ were genotyped with these markers (Table 2).

Marker associations for ADFI and ADG are presented in Table 2. A Bonferroni correction for multiple testing was applied to the $P$-values obtained from the genotyping analysis (Table 2). $P$-values were multiplied by the number of markers tested $(n=47)$ to obtain a corrected $P$. After correction, 16 markers between 38.16 and 38.37 $\mathrm{Mb}$ were significantly associated with ADFI $(P<0.05)$ and 20 markers between 38.16 and $38.41 \mathrm{Mb}$ were associated with ADG $(P<0.05)$.

Two markers within the NCAPG gene locus were associated (after multiple correction) with ADFI $(P \leq$ $0.02)$ and seven were associated with ADG $(P=0.003$; Table 2); one (83714_224) was the same marker previously identified by Setoguchi et al. [8] (p.I442M). However, several other markers were identified with lower $P$-values for ADFI and ADG (Table 2).

To determine whether these markers may adversely affect carcass and meat traits, they were analyzed for association with hot carcass weight (HCW), adjusted fat thickness (AFT), marbling score (MARB), ribeye area (REA), and SSF. The phenotypic data is presented in Table 3 and includes: number of animals, mean with standard deviation, and range of phenotypes. The effects and association between meat quality and carcass traits for the markers on BTA6 are presented in Table 4. The alleles for positive effects or increases in ADFI and ADG were associated with positive effects or higher HCW and REA. These same alleles had negative effects or lower AFT. Marbling and SSF were not significant for any of the markers tested (Table 4).

\section{Validation population analysis}

Twenty of the markers genotyped in the discovery population were genotyped in a validation population of crossbred animals $(n=406$; Table 5$)$. This set of markers included two markers in the LAP3 gene locus, 6 in NCAPG and 9 in the LCORL gene loci. Of the 20 markers, 13 and 17 were associated with ADFI and ADG, respectively, after Bonferroni correction in the discovery population. In addition to these 20 markers, six markers 
Table 1 SNP identified in LAP3, LCORL, and NCAPG genes in crossbred steers.

\begin{tabular}{|c|c|c|c|c|c|c|c|c|c|c|c|}
\hline Gene $^{1}$ & $\begin{array}{l}\text { Marker } \\
\text { Name }^{2}\end{array}$ & $\begin{array}{c}\text { Accession } \\
\text { Number }\end{array}$ & $\begin{array}{c}\text { dbSTS } \\
\text { Id }\end{array}$ & ss \# & $\mathrm{SNP}^{3}$ & $\begin{array}{l}\text { Position in } \\
\text { Gene }^{4}\end{array}$ & $\begin{array}{l}\text { Location } \\
\text { on BTA6 }{ }^{5}\end{array}$ & $\begin{array}{l}\text { For Primer } \\
\text { Name }\end{array}$ & For Primer Seq & $\begin{array}{l}\text { Rev Primer } \\
\text { Name }\end{array}$ & Rev Primer Seq \\
\hline LAP3 & 79140_312 & GF102128 & 1233209 & 196003657 & INDEL & p.E47del & 37964162 & LAP3-EX2F & GCCTTGTTCTTTTGTCCTTGAG & LAP3-EX2R & TTGGGTTGAAGTAGCCTGATTT \\
\hline$\angle A P 3$ & 79144_139 & GF102139 & 1233220 & 196003658 & S & p.D92E & 37966065 & LAP3-EX4F & ACCAAATTTCTTGGGGAGTTTT & LAP3-EX4R & AACACTGCAAGAGGGAACAAAT \\
\hline LAP3 & 79146_218 & GF102161 & 1233242 & 196003659 & Y & c.504C>T & 37967629 & LAP3-EX5F & GGGAAGAAGTGAAAGCTCAGAA & LAP3-EX5R & AAACTGACTAGGGCCATGAGAG \\
\hline LAP3 & 79150_94 & GF102129 & 1233210 & 196003660 & Y & $\mathrm{C} .834 \mathrm{C}>\mathrm{T}$ & 37971945 & LAP3-EX7F & CATTTTCATTTCCTGCCTTGT & LAP3-EX7R & GTAAGCAAAGGGAAGACAGCAT \\
\hline LAP3 & 79152_265 & GF102108 & 1233189 & 196003661 & $Y$ & & 37977863 & LAP3-EX8F & TGGAAATTTCTTGGTGCTTGTA & LAP3-EX8R & CTGACTGCTAACCACATTTTGC \\
\hline LAP3 & 79154_194 & GF102068 & 1233149 & 196003662 & $\mathrm{Y}$ & & 37979109 & LAP3-EX9F & САСАСТCCATGTTCCTTCATGT & LAP3-EX9R & TGCAGGACTTGTTTTTCTTCTCA \\
\hline LAP3 & 79154_347 & GF102068 & 1233149 & 196003663 & $S$ & & 37979262 & LAP3-EX9F & CACACTCCATGTTCCTTCATGT & LAP3-EX9R & TGCAGGACTTGTTTTCTTCTCA \\
\hline LAP3 & 79158_103 & GF102082 & 1233163 & 196003664 & $s$ & & 37984197 & LAP3-EX11F & AGGATTTGCCAACATCATCTTT & LAP3-EX11R & СCATGCCTTCTTTTACAAAGCTA \\
\hline LAP3 & 79158_104 & GF102082 & 1233163 & 196003665 & $\mathbf{R}$ & & 37984198 & LAP3-EX11F & AGGATTTGCCAACATCATCTTT & LAP3-EX11R & CCATGCCTTCTTTTACAAAGCTA \\
\hline LAP3 & 79160_105 & GF102096 & 1233177 & 196003666 & $\mathrm{Y}$ & & 37985986 & LAP3-EX12F & ATTTCAGCAAATGCCCTACAAT & LAP3-EX12R & TTGAAAATGCAAAAACCAGTTG \\
\hline LAP3 & 79162_241 & GF102069 & 1233150 & 196003667 & K & $\begin{array}{l}\text { c. } \\
{ }^{*} 1641 T>G\end{array}$ & 37986804 & LAP3-EX13F & TGGGGTTAGATGTTGATTTTTTG & LAP3-EX13R & GTGAATATGAGAGCCACACCAG \\
\hline LAP3 & $79162 \_246$ & GF102069 & 1233150 & 196003668 & $Y$ & $\begin{array}{l}\text { c. } \\
* 1646 C>T\end{array}$ & 37986809 & LAP3-EX13F & TGGGGTTAGATGTTGATTTTTG & LAP3-EX13R & GTGAATATGAGAGCCACACCAG \\
\hline$\angle A P 3$ & 79162_438 & GF102069 & 1233150 & 196003669 & $\mathrm{R}$ & $\begin{array}{l}\text { C. } \\
* 1838 A>G\end{array}$ & 37987001 & LAP3-EX13F & TGGGGTTAGATGTTGATTITTG & LAP3-EX13R & GTGAATATGAGAGCCACACCAG \\
\hline NCAPG & 83704_234 & GF110810 & 1241907 & 263198259 & $\mathrm{R}$ & & 38157198 & $\begin{array}{l}\text { NCAPG- } \\
\text { EX4-F }\end{array}$ & TCTGTCCTTTTCAAGGGATGTT & $\begin{array}{l}\text { NCAPG- } \\
\text { EX4-R }\end{array}$ & CAGCCACACAATAATGCGATAG \\
\hline NCAPG & 83704_335 & GF110810 & 1241907 & 263198262 & S & & 38157299 & $\begin{array}{l}\text { NCAPG-EX4- } \\
F\end{array}$ & TCTGTCCTTITCAAGGGATGTT & $\begin{array}{l}\text { NCAPG-EX4- } \\
R\end{array}$ & CAGCCACACAATAATGCGATAG \\
\hline NCAPG & 83704_358 & GF110810 & 1241907 & 263198260 & W & & 38157322 & $\begin{array}{l}\text { NCAPG-EX4- } \\
F\end{array}$ & TCTGTCCTTTCAAGGGATGTT & $\begin{array}{l}\text { NCAPG-EX4- } \\
R\end{array}$ & CAGCCACACAATAATGCGATAG \\
\hline NCAPG & 83704_366 & GF110810 & 1241907 & 263198261 & $\mathbf{R}$ & & 38157330 & $\begin{array}{l}\text { NCAPG- } \\
\text { EX4-F }\end{array}$ & TCTGTCCTTTTCAAGGGATGTT & $\begin{array}{l}\text { NCAPG- } \\
\text { EX4-R }\end{array}$ & CAGCCACACAATAATGCGATAG \\
\hline NCAPG & 83708_361 & GF110811 & 1241908 & 263198263 & $\mathrm{Y}$ & & 38158932 & $\begin{array}{l}\text { NCAPG- } \\
\text { EX6-F }\end{array}$ & GATGGAAGCTAGAGGGGAACTT & $\begin{array}{l}\text { NCAPG- } \\
\text { EX6-R }\end{array}$ & CTTTTCATGGTAGGCCTAATGC \\
\hline NCAPG & 83712_168 & GF110812 & 1241909 & 263198264 & $\mathbf{R}$ & c. $1261 \mathrm{~A}>\mathrm{G}$ & 38163729 & $\begin{array}{l}\text { NCAPG- } \\
\text { EX8-F }\end{array}$ & TTCTCACTTAATGGGGAGCTGT & $\begin{array}{l}\text { NCAPG- } \\
\text { EX8-R }\end{array}$ & AAATTTTAAGCAAAAACCTGCAA \\
\hline NCAPG & $83712 \_422$ & GF110812 & 1241909 & 263198265 & $\mathrm{R}$ & & 38163983 & $\begin{array}{l}\text { NCAPG-EX8- } \\
F\end{array}$ & TTCTCACTTAATGGGGAGCTGT & $\begin{array}{l}\text { NCAPG-EX8- } \\
R\end{array}$ & AAATTTAAGCAAAAACCTGCAA \\
\hline NCAPG & 83714_209 & GF110813 & 1241910 & 263198266 & M & C. $1357 \mathrm{~A}>\mathrm{C}$ & 38164388 & $\begin{array}{l}\text { NCAPG-EX9- } \\
F\end{array}$ & TTTCAGAATGTGAATTITGGCTTA & $\begin{array}{l}\text { NCAPG-EX9- } \\
R\end{array}$ & GTGTGAAAGGGACTGGGTTTIA \\
\hline NCAPG & 83714_224 & GF110813 & 1241910 & 263198267 & K & p.I442M & 38164403 & $\begin{array}{l}\text { NCAPG- } \\
\text { EX9-F }\end{array}$ & TTTCAGAATGTGAATTTTGGCTTA & $\begin{array}{l}\text { NCAPG- } \\
\text { EX9-R }\end{array}$ & GTGTGAAAGGGACTGGGTTTTA \\
\hline NCAPG & 83724_658 & GF110818 & 1241915 & 263198273 & $\mathrm{Y}$ & c. $2158 \mathrm{~T}>\mathrm{C}$ & 38180790 & $\begin{array}{l}\text { NCAPG- } \\
\text { EX14-F }\end{array}$ & ATTTACTTTTGGTGGGGGATGT & $\begin{array}{l}\text { NCAPG- } \\
\text { EX14-R }\end{array}$ & САAССTCCCAATAGTCATTTCA \\
\hline NCAPG & 83726_315 & GF110815 & 1241912 & 263198269 & R & & 38192653 & $\begin{array}{l}\text { NCAPG- } \\
\text { EX15-F }\end{array}$ & ATGTAAGGCCTAAAAGGGAGGT & $\begin{array}{l}\text { NCAPG- } \\
\text { EX15-R }\end{array}$ & GGAAAGAGCCAAATAATTCAGG \\
\hline NCAPG & 83730_778 & GF110816 & 1241913 & 263198270 & M & p.L877M & 38195339 & $\begin{array}{l}\text { NCAPG- } \\
\text { EX17-F }\end{array}$ & GAGCGTGAATGGTTCAGTACAC & $\begin{array}{l}\text { NCAPG- } \\
\text { EX17-R }\end{array}$ & ACTTCAAGAAATGAGCACCAAA \\
\hline NCAPG & 83732_316 & GF110819 & 1241916 & 263198271 & $\mathbf{R}$ & & 38195743 & $\begin{array}{l}\text { NCAPG- } \\
\text { EX18-F }\end{array}$ & AGTATTTGGTGCTCATTTCTTGA & $\begin{array}{l}\text { NCAPG- } \\
\text { EX18-R }\end{array}$ & TCCCAAGTGAATTTAATTAGAAAAA \\
\hline
\end{tabular}


Table 1 SNP identified in LAP3, LCORL, and NCAPG genes in crossbred steers. (Continued)

\begin{tabular}{|c|c|c|c|c|c|c|c|c|c|c|}
\hline LCORL & 79205_443 & GF102113 & 1233194 & 196003679 & $\mathrm{R}$ & 38233294 & $\begin{array}{l}\text { LOC540095- } \\
\text { EX6F }\end{array}$ & CCTATGTAGTGCCTTCCCAGTC & $\begin{array}{l}\text { LOC540095- } \\
\text { EX6R }\end{array}$ & CTCGTCCTGCTTCTTAGTTTGT \\
\hline LCORL & 81441_243 & GF102084 & 1233165 & 196003694 & $\mathrm{Y}$ & 38255270 & $\begin{array}{l}\text { LOC540095- } \\
\text { IN4.4F }\end{array}$ & GCATGAATGACAAAACTGTGCT & $\begin{array}{l}\text { LOC540095- } \\
\text { IN4.4R }\end{array}$ & САTTTTGCCCTTAAGCCTTCTA \\
\hline LCORL & 81439_210 & GF102101 & 1233182 & 196003693 & $\mathrm{R}$ & 38257174 & $\begin{array}{l}\text { LOC540095- } \\
\text { IN4.3F }\end{array}$ & TTGCCTTCAGTTCTCTTAGGC & $\begin{array}{l}\text { LOC540095- } \\
\text { IN4.3R }\end{array}$ & TTGCAAAATTATGGCATTTCAC \\
\hline LCORL & 81435_188 & GF102143 & 1233224 & 196003692 & $\mathrm{Y}$ & 38284737 & $\begin{array}{l}\text { LOC540095- } \\
\text { IN4.1F }\end{array}$ & TAGCCTGACTGCATCCATCTAA & $\begin{array}{l}\text { LOC540095- } \\
\text { IN4.1R }\end{array}$ & GGAAATCCCTGGTTAAGAATCC \\
\hline LCORL & 79197_655 & GF110814 & 1241911 & 263198268 & $\mathrm{R}$ & 38327100 & $\begin{array}{l}\text { LOC540095- } \\
\text { EX2F }\end{array}$ & TCTCACGTAGAGTGTATGGATAAGC & $\begin{array}{l}\text { LOC540095- } \\
\text { EX2R }\end{array}$ & GAGTTCCAGGCTGCCTATATCA \\
\hline LCORL & 81433_176 & GF110817 & 1241914 & 263198272 & Y & 38314844 & $\begin{array}{l}\text { LOC540095- } \\
\text { IN3.1F }\end{array}$ & GCAGGTGAAAATCCCAATACAC & $\begin{array}{l}\text { LOC540095- } \\
\text { IN3.1R }\end{array}$ & GGGCCAAACTAGCCTTATTTCT \\
\hline LCORL & 81419_461 & GF102100 & 1233181 & 196003691 & M & 38342145 & $\begin{array}{l}\text { LOC540095- } \\
\text { IN1.9F }\end{array}$ & GACTTCAAATTTTTGCCCAGAG & $\begin{array}{l}\text { LOC540095- } \\
\text { IN1.9R }\end{array}$ & GGTGTTCTTACCCTGTCTCAGC \\
\hline LCORL & 81413_159 & GF102115 & 1233196 & 196003686 & S & 38359337 & $\begin{array}{l}\text { LOC540095- } \\
\text { IN1.6F }\end{array}$ & AGGATCAACCATTAGGATGTGC & $\begin{array}{l}\text { LOC540095- } \\
\text { IN1.6R }\end{array}$ & AACTGGGAAGAGAGCAAGTGAG \\
\hline LCORL & 81413_221 & GF102115 & 1233196 & 196003684 & W & 38359399 & $\begin{array}{l}\text { LOC540095- } \\
\text { IN1.6F }\end{array}$ & AGGATCAACCATTAGGATGTGC & $\begin{array}{l}\text { LOC540095- } \\
\text { IN1.6R }\end{array}$ & AACTGGGAAGAGAGCAAGTGAG \\
\hline LCORL & 81413_226 & GF102115 & 1233196 & 196003687 & W & 38359404 & $\begin{array}{l}\text { LOC540095- } \\
\text { IN1.6F }\end{array}$ & AGGATCAACCATTAGGATGTGC & $\begin{array}{l}\text { LOC540095- } \\
\text { IN1.6R }\end{array}$ & AACTGGGAAGAGAGCAAGTGAG \\
\hline LCORL & 81413_231 & GF102115 & 1233196 & 196003689 & M & 38359409 & $\begin{array}{l}\text { LOC540095- } \\
\text { IN1.6F }\end{array}$ & AGGATCAACCATTAGGATGTGC & $\begin{array}{l}\text { LOC540095- } \\
\text { IN1.6R }\end{array}$ & AACTGGGAAGAGAGCAAGTGAG \\
\hline LCORL & 81413_238 & GF102115 & 1233196 & 196003690 & W & 38359416 & $\begin{array}{l}\text { LOC540095- } \\
\text { IN1.6F }\end{array}$ & AGGATCAACCATTAGGATGTGC & $\begin{array}{l}\text { LOC540095- } \\
\text { IN1.6R }\end{array}$ & AACTGGGAAGAGAGCAAGTGAG \\
\hline LCORL & 81405_282 & GF102099 & 1233180 & 196003685 & M & 38376731 & $\begin{array}{l}\text { LOC540095- } \\
\text { IN1.2F }\end{array}$ & TCGGGTCCTCTTTTACTGTCAT & $\begin{array}{l}\text { LOC540095- } \\
\text { IN1.2R }\end{array}$ & СТTACCACGATCTCCTTTCCAC \\
\hline
\end{tabular}

SNP in bold were genotyped using the Sequenom MassArray System.

${ }^{2}$ Marker name in USMARC database.

${ }^{3}$ IUB Codes for $S N P$ are $K=G / T, M=A / C, R=A / G, S=C / G, W=A / T, Y=C / T$.

${ }^{4}$ Position based on NM_174098 Bos taurus leucine aminopeptidase 3 (LAP3) mRNA; NM_001102376 Bos taurus non-SMC condensin I complex, subunit G (NCAPG), mRNA; and NM_001192357 Bos taurus ligand dependent nuclear receptor corepressor-like (LCORL) mRNA.

${ }^{5}$ Position based on the Btau 4.0 genome assembly. 
Table 2 SNP marker associations and estimated effects for ADFI and ADG in the discovery population of crossbred steers.

\begin{tabular}{|c|c|c|c|c|c|c|c|c|c|c|c|}
\hline \multirow[b]{2}{*}{ Marker $^{1}$} & \multirow[b]{2}{*}{ Gene $^{2}$} & \multirow[b]{2}{*}{ Position $^{3}$} & \multirow[b]{2}{*}{ IUB } & \multirow[b]{2}{*}{$\begin{array}{c}\# \\
\text { Genotypes }^{4} \\
\end{array}$} & \multirow[b]{2}{*}{ MAF } & \multicolumn{3}{|c|}{$\begin{array}{l}\text { Average Daily Feed Intake } \\
\text { (ADFI) }\end{array}$} & \multicolumn{3}{|c|}{ Average Daily Gain (ADG) } \\
\hline & & & & & & Effect $^{5}$ & $\begin{array}{c}P- \\
\text { value }\end{array}$ & Correction $^{6}$ & Effect & $\begin{array}{c}P- \\
\text { value }\end{array}$ & Correction \\
\hline ARS-BFGL-NGS-77584 & & 37855236 & $\mathrm{R}$ & 1182 & $G=0.10$ & -0.026 & 0.8 & NS & -0.0078 & 0.7 & NS \\
\hline After2Run10KSet7323 & & 37856744 & Y & 1075 & $\mathrm{~T}=0.30$ & -0.14 & 0.01 & NS & -0.029 & 0.01 & NS \\
\hline Hapmap31601-BTC-034240 & & 37859827 & $\mathrm{R}$ & 1076 & $G=0.28$ & -0.15 & 0.005 & NS & -0.033 & 0.004 & NS \\
\hline Hapmap28705-BTC-034230 & & 37862818 & s & 1090 & $G=0.31$ & -0.14 & 0.010 & NS & -0.033 & 0.003 & NS \\
\hline Hapmap25169-BTC-034210 & & 37870369 & $\mathrm{R}$ & 1186 & $A=0.30$ & -0.15 & 0.006 & NS & -0.033 & 0.003 & NS \\
\hline Hapmap30444-BTC-052947 & & 37875093 & Y & 1164 & $C=0.49$ & 0.16 & 0.004 & NS & 0.036 & 0.002 & NS \\
\hline After2Run10KSet5598 & & 37875791 & Y & 1186 & $C=0.30$ & -0.14 & 0.01 & NS & -0.032 & 0.004 & NS \\
\hline Hapmap32743-BTC-052998 & & 37879284 & $\mathrm{R}$ & 1011 & $A=0.23$ & -0.073 & 0.2 & NS & -0.024 & 0.04 & NS \\
\hline Hapmap23977-BTC-053017 & & 37880952 & M & 1176 & $A=0.30$ & -0.15 & 0.006 & NS & -0.032 & 0.005 & NS \\
\hline 79140_312 & LAP3 & 37964162 & INDEL & 1165 & $\begin{array}{c}\mathrm{DEL}= \\
0.09\end{array}$ & 0.11 & 0.2 & NS & -0.013 & 0.5 & NS \\
\hline Hapmap56977-ss46527055 & LAP3 & 37967630 & R & 1192 & $A=0.13$ & -0.051 & 0.5 & NS & -0.013 & 0.4 & NS \\
\hline 79152_265 & LAP3 & 37977863 & Y & 1181 & $C=0.33$ & -0.020 & 0.7 & NS & -0.021 & 0.05 & NS \\
\hline 79154_194 & LAP3 & 37979109 & Y & 1078 & $\mathrm{~T}=0.38$ & -0.0072 & 0.9 & NS & -0.021 & 0.06 & NS \\
\hline 79154_347 & $\angle A P 3$ & 37979262 & S & 1098 & $C=0.34$ & -0.022 & 0.7 & NS & -0.021 & 0.04 & NS \\
\hline 79158_103 & LAP3 & 37984197 & S & 1193 & $G=0.34$ & 0.026 & 0.6 & NS & 0.021 & 0.05 & NS \\
\hline 79158_104 & LAP3 & 37984198 & $\mathrm{R}$ & 1190 & $G=0.33$ & -0.021 & 0.7 & NS & -0.020 & 0.07 & NS \\
\hline 79160_105 & LAP3 & 37985986 & Y & 1127 & $\mathrm{~T}=0.34$ & -0.0086 & 0.9 & NS & -0.019 & 0.08 & NS \\
\hline 79162_241 & LAP3 & 37986804 & K & 1124 & $\mathrm{~T}=0.37$ & -0.0064 & 0.9 & NS & -0.22 & 0.05 & NS \\
\hline 79162_246 & LAP3 & 37986809 & Y & 1197 & $C=0.34$ & -0.020 & 0.7 & NS & -0.21 & 0.04 & NS \\
\hline ARS-BFGL-NGS-44699 & & 38011603 & S & 1183 & $C=0.10$ & -0.085 & 0.3 & NS & -0.021 & 0.2 & NS \\
\hline BTA-100891 & & 38076964 & Y & 1063 & $T=0.26$ & -0.083 & 0.1 & NS & -0.028 & 0.008 & NS \\
\hline 83704_234 & NCAPG & 38157198 & $\mathrm{R}$ & 1085 & $A=0.42$ & 0.18 & 0.0006 & 0.03 & 0.046 & 2.E-05 & 0.0009 \\
\hline 83704_366 & NCAPG & 38157330 & $\mathrm{R}$ & 1187 & $G=0.12$ & -0.11 & 0.2 & NS & -0.013 & 0.4 & NS \\
\hline 83708_361 & NCAPG & 38158932 & Y & 1092 & $C=0.41$ & 0.17 & 0.001 & 0.05 & 0.045 & 4.E-05 & 0.002 \\
\hline $83712 \_168^{7}$ & NCAPG & 38163729 & $\mathrm{R}$ & 1004 & $G=0.47$ & -0.16 & 0.003 & NS & -0.045 & 9.E-05 & 0.004 \\
\hline 83714_224 & NCAPG & 38164403 & K & 1132 & $G=0.34$ & 0.21 & 8.E-05 & 0.004 & 0.059 & 1.E-07 & 5.E-06 \\
\hline 83724_658 & NCAPG & 38180790 & Y & 1100 & $C=0.41$ & 0.17 & 0.001 & 0.05 & 0.045 & 5.E-05 & 0.002 \\
\hline 83730_778 & NCAPG & 38195339 & M & 1022 & $A=0.42$ & 0.17 & 0.003 & NS & 0.058 & 1.E-06 & 0.00005 \\
\hline 83732_316 & NCAPG & 38195743 & $\mathrm{R}$ & 1050 & $G=0.42$ & 0.14 & 0.008 & NS & 0.044 & 1.E-04 & 0.005 \\
\hline Hapmap24122-BTC-041153 & & 38217816 & K & 1193 & $G=0.35$ & 0.22 & 1.E-05 & 0.0005 & 0.059 & 2.E-08 & 9.E-07 \\
\hline Hapmap24041-BTC-041151 & & 38220879 & K & 1192 & $\mathrm{~T}=0.35$ & 0.22 & 1.E-05 & 0.0005 & 0.059 & 2.E-08 & 9.E-07 \\
\hline Hapmap31609-BTC-041148 & & 38223329 & $\mathrm{R}$ & 1192 & $A=0.38$ & 0.17 & 0.0005 & 0.02 & 0.045 & 1.E-05 & 0.0005 \\
\hline Hapmap28154-BTC-0411348 & LCORL & 38228679 & $\mathrm{R}$ & 935 & $C=0.38$ & 0.18 & 0.0003 & 0.01 & 0.045 & 2.E-05 & 0.0009 \\
\hline Hapmap27082-BTC-041131 & LCORL & 38233962 & $\mathrm{R}$ & 1121 & $G=0.37$ & 0.18 & 0.0002 & 0.009 & 0.047 & 4.E-06 & 0.0002 \\
\hline Hapmap26855-BTC-041123 & LCORL & 38239191 & $\mathrm{R}$ & 1075 & $G=0.33$ & 0.21 & 8.E-05 & 0.004 & 0.058 & 1.E-07 & 5.E-06 \\
\hline Hapmap23341-BTC-041113 & LCORL & 38250098 & K & 1161 & $T=0.36$ & 0.21 & 4.E-05 & 0.002 & 0.056 & 1.E-07 & 5.E-06 \\
\hline 81441_243 & LCORL & 38255270 & $S$ & 822 & $G=0.28$ & -0.24 & 5.E-06 & 0.0002 & -0.058 & 2.E-07 & $9 \mathrm{E}-06$ \\
\hline 81435_188 & LCORL & 38284737 & Y & 892 & $C=0.38$ & 0.17 & 0.0004 & 0.02 & 0.042 & 3.E-05 & 0.001 \\
\hline $\begin{array}{l}\text { Hapmap28589-BTC- } \\
041045^{9}\end{array}$ & LCORL & 38300298 & $S$ & 1190 & $G=0.33$ & 0.22 & 2.E-05 & 0.0009 & 0.061 & $1 . E-08$ & 5.E-07 \\
\hline Hapmap33629-BTC-041038 & LCORL & 38313142 & Y & 999 & $\mathrm{~T}=0.30$ & 0.22 & 2.E-05 & 0.0009 & 0.059 & 5.E-08 & 2.E-06 \\
\hline Hapmap28835-BTC-041022 & LCORL & 38326993 & $S$ & 1061 & $G=0.18$ & -0.12 & 0.07 & NS & -0.027 & 0.04 & NS \\
\hline 81419_461 & LCORL & 38342145 & M & 1058 & $C=0.05$ & -0.17 & 0.1 & NS & -0.046 & 0.04 & NS \\
\hline Hapmap33694-BTC-041005 & LCORL & 38342183 & $\mathrm{R}$ & 1190 & $G=0.16$ & -0.10 & 0.1 & NS & -0.038 & 0.005 & NS \\
\hline $81405 \_282^{9}$ & LCORL & 38376731 & $M$ & 1196 & $A=0.33$ & 0.22 & 2.E-05 & 0.0009 & 0.061 & $1 . \mathrm{E}-08$ & 5.E-07 \\
\hline Hapmap33650-BTC-073421 & & 38409471 & Y & 1041 & $C=0.40$ & -0.14 & 0.004 & NS & -0.036 & 0.0003 & 0.01 \\
\hline Hapmap31943-BTC-050642 & & 38427989 & Y & 1181 & $C=0.08$ & -0.11 & 0.2 & NS & -0.039 & 0.02 & NS \\
\hline
\end{tabular}


Table 2 SNP marker associations and estimated effects for ADFI and ADG in the discovery population of crossbred steers. (Continued)

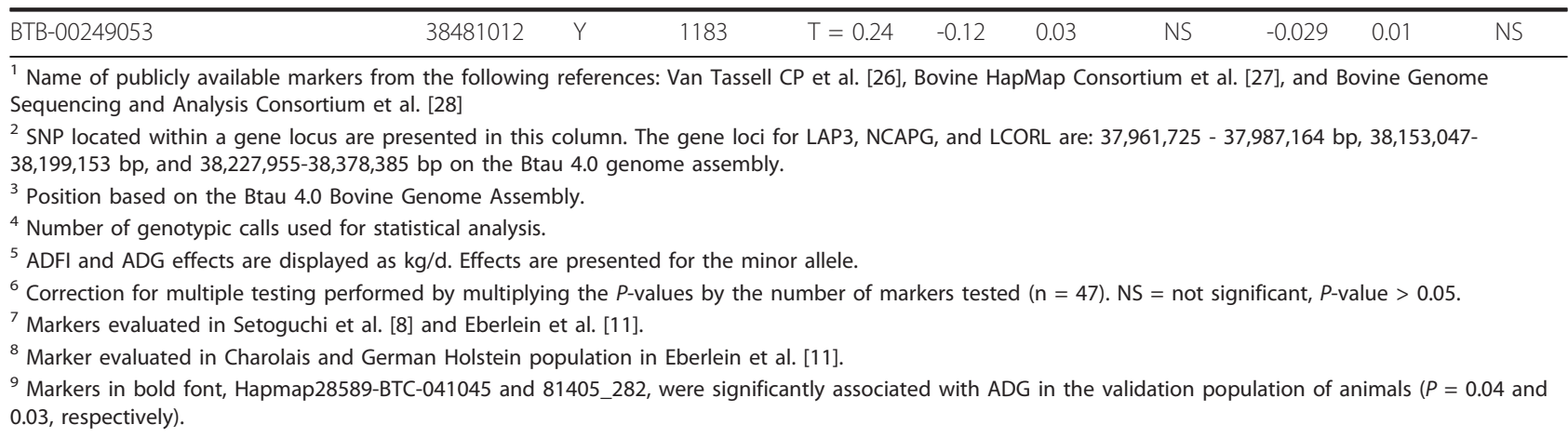

from the Illumina BovineSNP50 BeadChip that were nominally significant ADG (4 were also nominally significant for ADFI) in the discovery population were also genotyped in the validation population of animals. Association analysis produced two markers (Hapmap28589BTC-041045, $P=0.04$ and $81405 \_282, P=0.03$ ) that were associated with ADG in these animals (Table 5). Both markers are located within the $L C O R L$ gene locus. Marker 83714_224 (or p.I422M) [8], in NCAPG was not significant in this population $(P=0.09)$. Genotypes were analyzed with Haploview for linkage disequilibrium (LD) in this population of animals (Figure 1). The LD between marker 83714_224 in NCAPG and 81405_282 in LCORL was lower at $r^{2}=0.89$ than the LD between these two markers in the SNP discovery population $\left(\mathrm{r}^{2}\right.$ $=0.96$ ) (Figure 2).

The two markers significantly associated with ADG in the validation population, Hapmap28589-BTC-041045 and $81405 \_282$, are each responsible for $1.6 \%$ of the additive phenotypic variation of ADG (data not shown); however, they are in complete LD (Figure 1). These same markers each account for $3.6 \%$ of the phenotypic variation of ADG in the discovery population. These markers also represent $2 \%$ of the ADFI phenotypic variation in the discovery population of animals. These markers are also in complete LD in the discovery population of animals (Figure 2).

Table 3 Description of the USMARC resource population carcass and meat quality phenotypic means.

\begin{tabular}{llll}
\hline Phenotype $^{\mathbf{1}}$ & $\mathbf{N}$ & Mean $^{\mathbf{2}}$ & Range \\
\hline Hot Carcass Weight $(\mathrm{kg})$ & 1066 & $353 \pm 34.6$ & $246.8-468.6$ \\
Adjusted Fat Thickness $(\mathrm{cm})$ & 1066 & $1.27 \pm 0.5$ & $0.1-3.3$ \\
Marbling $(\mathrm{MSU})^{3}$ & 1066 & $531.4 \pm 91.8$ & $300-910$ \\
Ribeye Area $\left(\mathrm{cm}^{2}\right)$ & 1066 & $78.7 \pm 8.4$ & $56.1-116.8$ \\
Slice Shear Force $(\mathrm{kg})$ & 1066 & $12.4 \pm 3$ & $6.48-27.38$ \\
\hline
\end{tabular}

\footnotetext{
${ }^{1}$ Slice shear force was measured at $14 \mathrm{~d}$ postmortem.

${ }^{2}$ Mean and standard deviation for each phenotype.

${ }^{3} \mathrm{MSU}=$ Marbling score unit. $400=$ Slight $^{00}, 500=$ Small $^{00}, 600=$ Modest $^{00}$.
}

\section{Discussion}

A previous single nucleotide polymorphism association analysis identified a $570 \mathrm{~Kb}$ region on BTA6 from 37.96 to $38.53 \mathrm{Mb}$ containing eight SNP with significant association $(P \leq 0.003)$ with ADG and three SNP with significant association with ADFI $(P \leq 0.002)$ [9]. The three markers significant for ADFI were the same markers significant for ADG. The most significant marker in this region from Snelling et al. [9] for ADG was ARS-BFGLNGS-45457 $\left(P\right.$, nominal $\left.=4.8 \times 10^{-6}\right)$ and the most significant marker for ADFI was Hapmap31285-BTC$041097\left(P\right.$, nominal $\left.=8 \times 10^{-4}\right)$. Of the markers presented in this study, 14 are more significant for ADFI $\left(P\right.$, nominal $\left.=5 \times 10^{-4}\right)$ and 11 were more significant for $\operatorname{ADG}\left(P\right.$, nominal $\left.=4 \times 10^{-6}\right)$ than the markers on the BovineSNP50 BeadChip.

We chose to evaluate $L A P 3, N C A P G$ and $L C O R L$ as potential positional and functional candidate genes on bovine chromosome 6 for steer feed intake and gain. These genes were within the region that was identified as significant for ADFI and ADG from a previous association analysis [9]. None of the markers in LAP3 or in close proximity to $L A P 3$ were significant after correction for multiple testing suggesting that these SNP are not in high LD with the causative mutation and that this region is not likely to be contributing to variation in feed intake and gain in this population of cattle. Our data do, however, suggest that the more likely region responsible for variation in cattle feed intake and gain is further downstream in the NCAPG-LCORL locus.

NCAPG is a subunit of the condensin 1 complex that is involved in the condensation of chromosomes and also interacts with a DNA methyltransferase linking methylation and chromatin condensation; however, the molecular and cellular events that might specifically tie NCAPG to growth and appetite have not been identified.

$L C O R L$ encodes a transcription factor that is thought to function during spermatogenesis. Aside from the association of SNPs in LCORL with human height 
Table 4 Association and effects of SNP significant for ADG on BTA6 with meat quality and carcass traits in the discovery population of crossbred steers.

\begin{tabular}{|c|c|c|c|c|c|c|c|c|c|c|c|c|c|c|c|c|}
\hline \multirow[b]{2}{*}{ Marker Name ${ }^{2}$} & \multirow[b]{2}{*}{ Minor Allele } & \multicolumn{3}{|c|}{ Adjusted Fat Thickness(cm) } & \multicolumn{3}{|c|}{ Hot Carcass Weight (kg) } & \multicolumn{3}{|c|}{ Ribeye Area $\left(\mathrm{cm}^{2}\right)$} & \multicolumn{3}{|c|}{ Marbling (MSU) ${ }^{1}$} & \multicolumn{3}{|c|}{ Slice Shear Force } \\
\hline & & Effect $^{3}$ & SE & $P$ & Effect $^{3}$ & SE & $P$ & Effect $^{3}$ & SE & $P$ & Effect $^{3}$ & SE & $P$ & Effect $^{3}$ & SE & $P$ \\
\hline $83704 \_234^{4}$ & A & -0.102 & 0.0245 & $3.8 \mathrm{E}-05$ & 8.69 & 1.634 & $1.8 \mathrm{E}-07$ & 1.99 & 0.3714 & $1.4 \mathrm{E}-07$ & -2.45 & 4.073 & 0.55 & -0.060 & 0.149 & 0.69 \\
\hline 83708_361 & C & -0.095 & 0.0247 & 0.0001 & 7.84 & 1.648 & 2.7E-06 & 1.83 & 0.3748 & $1.4 \mathrm{E}-06$ & -2.86 & 4.110 & 0.49 & -0.081 & 0.151 & 0.59 \\
\hline $83712 \_168^{4}$ & G & -0.086 & 0.0263 & 0.001 & 8.05 & 1.753 & $5.9 \mathrm{E}-06$ & 1.83 & 0.3986 & $6.0 \mathrm{E}-06$ & -5.33 & 4.371 & 0.22 & -0.012 & 0.160 & 0.94 \\
\hline 83714_224 ${ }^{4}$ & G & -0.125 & 0.0250 & $9.2 \mathrm{E}-07$ & 10.39 & 1.671 & $1.3 \mathrm{E}-09$ & 2.33 & 0.3798 & $1.9 \mathrm{E}-09$ & -6.94 & 4.165 & 0.10 & -0.031 & 0.153 & 0.84 \\
\hline 83724_658 & C & -0.096 & 0.0250 & 0.0002 & 8.25 & 1.671 & $1.2 \mathrm{E}-06$ & 1.98 & 0.3801 & $3.1 \mathrm{E}-07$ & -4.23 & 4.168 & 0.31 & -0.073 & 0.153 & 0.63 \\
\hline 83730_778 & A & -0.100 & 0.0271 & 0.0002 & 9.15 & 1.807 & $6.2 \mathrm{E}-07$ & 2.20 & 0.4110 & $1.5 \mathrm{E}-07$ & -4.47 & 4.506 & 0.32 & -0.086 & 0.166 & 0.60 \\
\hline 83732_316 & G & -0.097 & 0.0256 & 0.0002 & 8.10 & 1.711 & $3.0 \mathrm{E}-06$ & 1.92 & 0.3890 & $1.2 \mathrm{E}-06$ & -3.27 & 4.266 & 0.44 & -0.084 & 0.157 & 0.59 \\
\hline Hapmap24122-BTC-041153 & G & -0.120 & 0.0236 & $5.3 \mathrm{E}-07$ & 10.56 & 1.577 & $7.4 \mathrm{E}-11$ & 2.43 & 0.3584 & $4.3 \mathrm{E}-11$ & -4.47 & 3.931 & 0.26 & -0.075 & 0.144 & 0.60 \\
\hline Hapmap24041-BTC-041151 & $\mathrm{T}$ & -0.119 & 0.0235 & $6.3 \mathrm{E}-07$ & 10.38 & 1.570 & $1.2 \mathrm{E}-10$ & 2.39 & 0.3569 & $6.9 \mathrm{E}-11$ & -3.83 & 3.914 & 0.33 & -0.085 & 0.143 & 0.55 \\
\hline Hapmap31609-BTC-041148 & $A$ & -0.108 & 0.0232 & 4.7E-06 & 8.56 & 1.552 & $6.3 \mathrm{E}-08$ & 2.02 & 0.3527 & $2.0 \mathrm{E}-08$ & -3.45 & 3.868 & 0.37 & -0.10 & 0.142 & 0.48 \\
\hline Hapmap28154-BTC-0411345 & C & -0.108 & 0.0235 & $5.4 \mathrm{E}-06$ & 8.31 & 1.568 & $1.9 \mathrm{E}-07$ & 1.87 & 0.3563 & $2.3 \mathrm{E}-07$ & -2.64 & 3.908 & 0.50 & -0.084 & 0.143 & 0.56 \\
\hline Hapmap27082-BTC-041131 & G & -0.108 & 0.0232 & $4.5 \mathrm{E}-06$ & 8.59 & 1.553 & $5.6 \mathrm{E}-08$ & 1.98 & 0.3527 & $3.6 \mathrm{E}-08$ & -3.93 & 3.869 & 0.31 & -0.091 & 0.142 & 0.52 \\
\hline Hapmap26855-BTC-041123 & G & -0.127 & 0.0244 & $2.8 \mathrm{E}-07$ & 10.41 & 1.631 & $4.9 \mathrm{E}-10$ & 2.12 & 0.3705 & $1.9 \mathrm{E}-08$ & -6.65 & 4.064 & 0.10 & -0.056 & 0.149 & 0.71 \\
\hline Hapmap23341-BTC-041113 & $\mathrm{T}$ & -0.114 & 0.0236 & $1.8 \mathrm{E}-06$ & 10.16 & 1.575 & $3.2 \mathrm{E}-10$ & 2.26 & 0.3579 & $6.7 \mathrm{E}-10$ & -4.34 & 3.925 & 0.27 & -0.077 & 0.144 & 0.59 \\
\hline 81441_243 & G & 0.112 & 0.0248 & $7.9 \mathrm{E}-06$ & -10.59 & 1.658 & 4.7E-10 & -2.25 & 0.3766 & $5.2 \mathrm{E}-09$ & 1.59 & 4.131 & 0.70 & 0.078 & 0.151 & 0.61 \\
\hline 81435_188 & C & -0.099 & 0.0231 & $2.2 \mathrm{E}-05$ & 8.23 & 1.545 & $1.7 \mathrm{E}-07$ & 1.71 & 0.3511 & $1.6 \mathrm{E}-06$ & -4.85 & 3.851 & 0.21 & -0.087 & 0.141 & 0.54 \\
\hline Hapmap28589-BTC-041045 & G & -0.130 & 0.0240 & $9.4 \mathrm{E}-08$ & 10.63 & 1.601 & $1.0 \mathrm{E}-10$ & 2.33 & 0.3640 & $4.3 \mathrm{E}-10$ & -5.86 & 3.992 & 0.14 & -0.044 & 0.146 & 0.77 \\
\hline Hapmap33629-BTC-041038 & $\mathrm{T}$ & -0.127 & 0.0245 & $3.6 \mathrm{E}-07$ & 10.30 & 1.639 & $8.4 \mathrm{E}-10$ & 2.10 & 0.3721 & $3.4 \mathrm{E}-08$ & -6.45 & 4.082 & 0.11 & -0.065 & 0.149 & 0.66 \\
\hline 81405_282 & A & -0.130 & 0.0242 & $1.4 \mathrm{E}-07$ & 10.84 & 1.619 & $7.2 \mathrm{E}-11$ & 2.38 & 0.3679 & $3.1 \mathrm{E}-10$ & -5.61 & 4.035 & 0.17 & -0.030 & 0.148 & 0.84 \\
\hline Hapmap33650-BTC-073421 & C & 0.054 & 0.0232 & 0.02 & -7.26 & 1.546 & 3.7E-06 & -1.35 & 0.3514 & 0.0001 & 5.01 & 3.854 & 0.19 & 0.043 & 0.141 & 0.76 \\
\hline
\end{tabular}

${ }^{1} \mathrm{MSU}=$ Marbling score unit. $400=$ Slight $^{00}, 500=$ Small $^{00}, 600=$ Modest $^{00}$

2 Name of publicly available markers from the following references: Van Tassell CP et al. [26], Bovine HapMap Consortium et al. [27], and Bovine Genome Sequencing and Analysis Consortium et al. [28].

${ }^{3}$ Effects of phenotypes are displayed in the following units: AFT $=\mathrm{cm}, \mathrm{HCW}=\mathrm{kg}, \mathrm{REA}=\mathrm{cm}^{2}$, Marbling $=\mathrm{MSU}$, and $\mathrm{SSF}=\mathrm{kg}$. The effects presented correspond to the minor allele.

${ }^{4}$ Markers evaluated in Setoguchi et al. [8] and Eberlein [11]

${ }^{5}$ Marker evaluated in Charolais and German Holstein population in Eberlein et al. [11]. 
Table 5 SNP marker associations and estimated effects for ADG in the validation population of crossbred steers ( $\mathrm{n}=$ 406).

\begin{tabular}{|c|c|c|c|c|c|c|c|c|}
\hline \multirow[b]{2}{*}{ Marker $^{1}$} & \multirow[b]{2}{*}{ Illumina BeadChip ${ }^{2}$} & \multirow[b]{2}{*}{ Rs\# } & \multirow[b]{2}{*}{ Position $^{3}$} & \multirow[b]{2}{*}{ IUB } & \multirow[b]{2}{*}{ MAF } & \multicolumn{3}{|c|}{ Average Daily Gain (ADG) } \\
\hline & & & & & & Effect $^{5}$ & $S E$ & $P$-value \\
\hline 79154_347 & & & 37979262 & S & $C=0.34$ & -0.016 & 0.0183 & 0.4 \\
\hline 79158_103 & & & 37984197 & S & $G=0.35$ & 0.019 & 0.0183 & 0.3 \\
\hline ARS-BGFL-NGS-45457 & $\checkmark$ & rs109447543 & 38102327 & W & $A=0.42$ & 0.029 & 0.0178 & 0.1 \\
\hline 83704_234 & & & 38157198 & R & $A=0.49$ & 0.018 & 0.0166 & 0.3 \\
\hline 83708_361 & & & 38158932 & Y & $C=0.49$ & -0.0015 & 0.0194 & 0.9 \\
\hline 83712_168 & & & 38163729 & $\mathrm{R}$ & $A=0.47$ & -0.0029 & 0.0186 & 0.9 \\
\hline 83714_224 & & & 38164403 & K & $G=0.42$ & 0.031 & 0.0179 & 0.09 \\
\hline 83724_658 & & & 38180790 & Y & $\mathrm{T}=0.49$ & -0.023 & 0.0171 & 0.2 \\
\hline 83732_316 & & & 38195743 & R & $G=0.49$ & 0.0042 & 0.0205 & 0.8 \\
\hline Hapmap24122-BTC-041153 & & & 38217816 & K & $G=0.43$ & 0.021 & 0.0175 & 0.2 \\
\hline Hapmap24041-BTC-041151 & & & 38220879 & K & $\mathrm{T}=0.44$ & 0.022 & 0.0175 & 0.2 \\
\hline Hapmap31609-BTC-041148 & & & 38223329 & $\mathrm{R}$ & $A=0.49$ & 0.015 & 0.0170 & 0.4 \\
\hline HapMap23507-BTC-041133 & $\checkmark$ & rs110961068 & 38233088 & M & $C=0.45$ & 0.00019 & 0.0192 & 1 \\
\hline Hapmap27082-BTC-041131 & & & 38233962 & R & $A=0.44$ & -0.027 & 0.0178 & 0.1 \\
\hline Hapmap26855-BTC-041123 & & & 38239191 & $\mathrm{R}$ & $G=0.42$ & 0.025 & 0.0185 & 0.2 \\
\hline Hapmap23341-BTC-041113 & & & 38250098 & K & $\mathrm{T}=0.44$ & 0.013 & 0.0181 & 0.5 \\
\hline $81441 \_243$ & & & 38255270 & S & $G=0.46$ & 0.017 & 0.0186 & 0.4 \\
\hline НарMар31285- BTC-041097 & $\checkmark$ & rs109294917 & 38256889 & Y & $C=0.44$ & 0.021 & 0.0175 & 0.2 \\
\hline 81435_188 & & & 38284737 & Y & $\mathrm{T}=0.42$ & -0.030 & 0.0189 & 0.1 \\
\hline Hapmap28589-BTC-041045 & & & 38300298 & 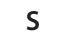 & $G=0.45$ & 0.038 & 0.0185 & 0.04 \\
\hline Hapmap33629-BTC-041038 & & & 38313142 & Y & $\mathrm{T}=0.44$ & 0.026 & 0.0191 & 0.2 \\
\hline Hapmap33694-BTC-041005 & & & 38342183 & $\mathrm{R}$ & $G=0.08$ & 0.021 & 0.0276 & 0.4 \\
\hline $81405 \_282^{6}$ & & & 38376731 & M & $A=0.46$ & 0.039 & 0.0180 & 0.03 \\
\hline HapMap27529-BTC-050639 & $\checkmark$ & rs110335851 & 38420476 & R & $G=0.29$ & -0.013 & 0.0187 & 0.5 \\
\hline HapMap28546- BTC-072715 & $\checkmark$ & rs43027303 & 38558526 & S & $G=0.21$ & -0.034 & 0.0204 & 0.1 \\
\hline HapMap27537- BTC-060891 & $\checkmark$ & rs110240246 & 38638962 & R & $A=0.40$ & -0.0064 & 0.0218 & 0.8 \\
\hline
\end{tabular}

${ }^{1}$ Name of publicly available markers from the following references: Van Tassell CP et al. [26], Bovine HapMap Consortium et al. [27], and Bovine Genome Sequencing and Analysis Consortium et al. [28].

${ }^{2}$ Markers with check marks are from the BovineSNP50 Illumina BeadChip. These markers were significantly associated with ADFI and/or ADG in the discovery population of crossbred steers [9].

${ }^{3}$ Position based on the Btau 4.0 Bovine Genome Assembly.

${ }^{4} \mathrm{NP}$ corresponding to the effects presented.

${ }^{5} \mathrm{ADFI}$ and $\mathrm{ADG}$ effects are displayed as $\mathrm{kg} / \mathrm{d}$. Effects are shown that correspond to the minor allele.

${ }^{6}$ Markers in bold font, Hapmap28589-BTC-041045 and 81405_282, were significantly associated with ADG in the validation population of animals $(P=0.04$ and 0.03 , respectively).

phenotypes, there is little published information regarding this gene and its potential functions relating to growth. While it is possible to speculate that as a transcription factor, SNPs that affect the transcription or translation of $L C O R L$ may result in increased or decreased regulation of gene(s) involved in growth and/ or appetite pathways, these functions have yet to be elucidated.

The NCAPG-LCORL locus has been interrogated in other populations of cattle for growth, meat quality and carcass traits. The most significant marker tested within the NCAPG-LCORL region in Japanese Black and Japanese Brown cattle for carcass weight, longissimus muscle area and subcutaneous fat thickness was the I422M marker (or marker 83714_224 in this study) in NCAPG
[8]. This same marker was significant for fetal growth in a crossbred population of Charolais and German Holstein [11]. In our crossbred steer population consisting of seven continental breeds of beef cattle, this marker was significant for similar traits including, HCW, REA, AFT, and also for ADG and ADFI; however, markers located further downstream and within the $L C O R L$ gene locus were even more significant. The only markers tested in all three of these studies were in the NCAPG gene. Only one marker within the $L C O R L$ gene locus (HapMap28154-BTC-041134) was tested in our population and in the study by Eberlein et al. [11], making a direct comparison of the $L C O R L$ region difficult. In our population of cattle, we detected 14 SNP in the NCAPG-LCORL locus that were significant for ADFI, 


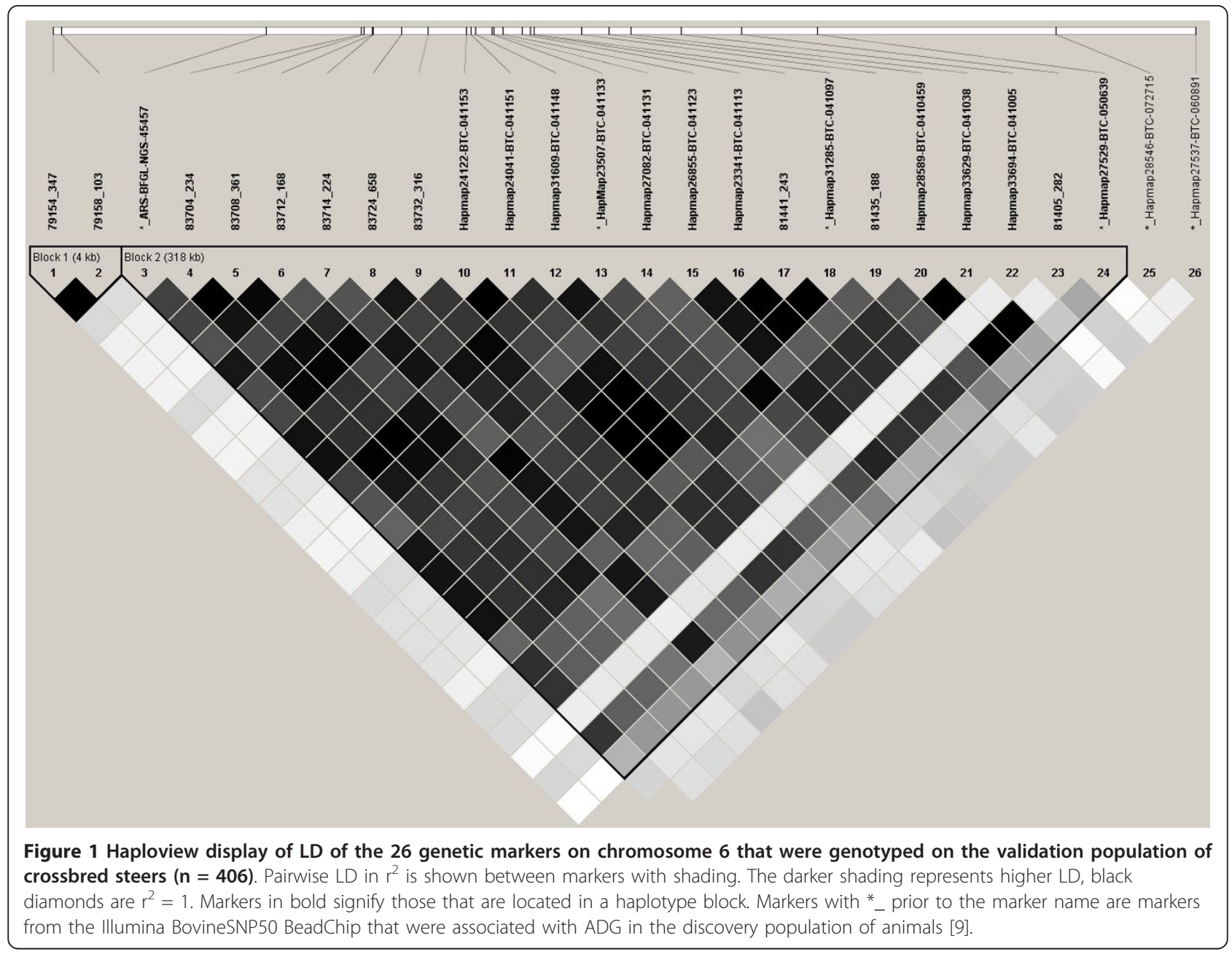

ADG and the carcass/meat quality traits $\mathrm{HCW}$, AFT and REA. All of these SNP are in LD $\left(r^{2} \leq 0.73\right)$, thus it is unclear which of these SNP and genes may be responsible for the phenotypic variation within this population of animals.

In an attempt to better define which SNPs are responsible for the phenotypic variation, we performed a validation of these markers in another crossbred population of animals consisting of 14 breeds of cattle. This produced two SNP associated with ADG located in the intronic regions of the $L C O R L$ gene and suggests that these SNP may be robust among differing breeds of cattle for the prediction of weight gain. The LD in the validation population of animals in this region differs from the LD in the discovery population of animals. An example is that the LD for the NCAPG SNP 83714_224 (I422M) marker and the most significant SNP for ADG in our study (HapMap28589-BTC041045) was $\mathrm{r}^{2}=0.96$ in our SNP discovery population and $r^{2}=0.91$ in the validation population.
The SNPs tested in our study and in previous work by Setoguchi et al. [8] are significant for traits that suggest they are involved in lean growth and fat deposition. In support of this, Gutiérrez-Gil et al. [2] also detected a QTL for subcutaneous fat in this region at BTA6:38.5, and McClure et al. [6] found a QTL for fat thickness at the $12^{\text {th }}$ rib at BTA6:36. These QTL plus the numerous QTL for growth and carcass weight seem to be harboring a gene or genes that are responsible for lean growth and fat deposition. Moreover, the NCAPG I442M SNP was associated with plasma arginine, symmetric dimethylarginine (SDMA), and linoleylcarnitine (C18:2) levels in a recent metabolomic study [29]. The allele that was associated with positive weight gain during 182-273 d (442M) was also associated with higher plasma levels of arginine, SDMA, and linoleylcarnitine [29]. The role of arginine in growth is through its activation of the mTOR pathway in skeletal muscle which increases protein synthesis $[29,30]$, and through its function as a precursor of nitrogen oxide which effects 


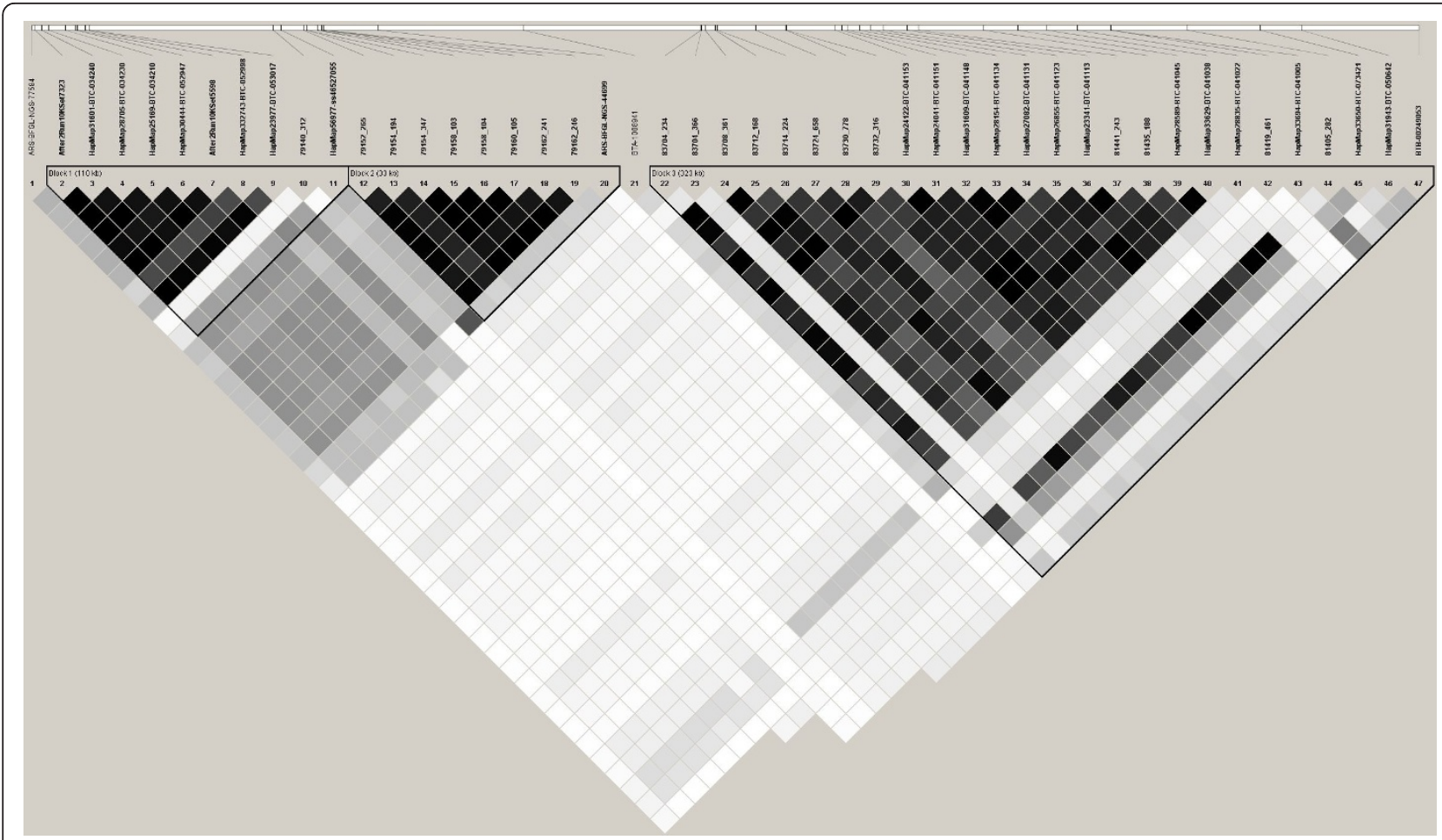

Figure 2 Haploview display of LD of the genetic markers on chromosome 6 genotyped on the SNP discovery population of crossbred steers $(\mathbf{n}=\mathbf{1}, 066)$. Pairwise $L D$ in $r^{2}$ is shown between markers by shading. The darker shading represents higher $L D$, black diamonds are $r^{2}=$ 1. Markers in bold signify those that are located in a haplotype block.

glucose uptake and insulin responsiveness in skeletal muscle $[29,31,32]$. These data suggest there is some type of physiological role for growth via arginine for the gene or genes located within this chromosomal region. As the gene functions for NCAPG and LCORL do not currently seem to suggest a direct interaction with the metabolites identified in Weikard et al. [29], and the SNP in these genes are in high LD with each other in our discovery and validation populations of animals, additional functional genomic studies will be required to elucidate the mechanisms of NCAPG and/or LCORL in growth.

\section{Conclusion}

In summary, we have identified several markers that are highly significant for steer feed intake and gain phentoypes. Moreover, several of the markers evaluated in this study are more significant for ADFI and ADG than the markers on the BovineSNP50 BeadChip. Genetic selection with these markers is likely to have an impact on HCW, REA and AFT with little influence on marbling or tenderness. It is not known how these markers will affect reproductive traits. These markers need to be evaluated on additional populations of animals to ensure that they maintain utility across breeds. We were able to confirm that two of these markers were significant for ADG in a validation population of crossbred cattle that included 14 breeds. These two markers may be particularly effective for the prediction of weight gain across various populations and breeds of cattle.

\section{Acknowledgements}

The authors would like to thank Linda Flathman for excellent technical assistance and Donna Griess for assistance with manuscript formatting and editing. Mention of trade name proprietary product or specified equipment does not constitute a guarantee or warranty by the USDA and does not imply approval to the exclusion of other products that may be suitable. USDA is an equal opportunity provider and employer.

\section{Author details}

${ }^{1}$ United States Department of Agriculture, Agricultural Research Service, U.S. Meat Animal Research Center, State Spur 18D, Clay Center, NE 68933, USA. ${ }^{2}$ Kansas State University, Manhattan, KS, USA. ${ }^{3}$ F\&J Livestock Consulting, LLC., Saronville, NE, USA.

\section{Authors' contributions}

ALP conceived of the study, carried out molecular genetic studies, and drafted the manuscript. AKS participated in the project design and carried out molecular genetic studies. LAK performed statistical analysis of genotypic data. WMS provided statistical advice. TPLS assisted with SNP selection. DAK, SDS and TLW managed meat quality and carcass phenotypic data collection. CLF, TGJ, HCF and LAK collected and provided feed efficiency phenotypic data. All authors read and approved the manuscript.

Received: 15 September 2011 Accepted: 14 December 2011 Published: 14 December 2011 


\section{References}

1. Nkrumah JD, Sherman EL, Li C, Marques E, Crews DH, Bartusiak R, Murdoch B, Wang Z, Basarab JA, Moore SS: Primary genome scan to identify putative quantitative trait loci for feedlot growth rate, feed intake, and feed efficiency of beef cattle. J Anim Sci 2007, 85:3170-3181.

2. Gutiérrez-Gil B, Williams JL, Homer D, Burton D, Haley CS, Wiener P: Search for quantitative trait loci affecting growth and carcass traits in a cross population of beef and dairy cattle. J Anim Sci 2009, 87:24-36.

3. Casas E, Shackelford SD, Keele JW, Stone RT, Kappes SM, Koohmaraie M: Quantitative trait loci affecting growth and carcass composition of cattle segregating alternate forms of myostatin. J Anim Sci 2000, 78:560-569.

4. Kneeland J, Li C, Basarab J, Snelling WM, Benkel B, Murdoch B, Hansen C, Moore SS: Identification and fine mapping of quantitative trait loci for growth traits on bovine chromosomes 2, 6, 14, 19, 21, 23 within one commercial line of Bos taurus. J Anim Sci 2004, 82:3405-3414.

5. Maltecca C, Weigel KA, Khatib H, Cowan M, Bagnato A: Whole-genome scan for quantitative trait loci associated with birth weight, gestation length and passive immune transfer in a Holstein $\times$ Jersey crossbred population. Anim Genet 2008, 40:27-34.

6. McClure MC, Morsci NS, Schnabel RD, Kim JW, Yao P, Rolf MM, McKay SD, Gregg SJ, Chapple RH, Northcutt SL, Taylor JF: A genome scan for quantitative trait loci influencing carcass, post-natal growth and reproductive traits in commercial Angus cattle. Anim Genet 2010, 41:597-607.

7. Takasuga A, Watanabe T, Mizoguchi Y, Hirano T, Ihara N, Takano A, Yokouchi K, Fujikawa A, Chiba K, Kobayashi N, Tatsuda K, Oe T, FurukawaKuroiwa M, Nishimura-Abe A, Fujita T, Inoue K, Mizoshita K, Ogino A, Sugimoto $Y$ : Identification of bovine QTL for growth and carcass traits in Japanese Black cattle by replication and identical-by-descent mapping. Mamm Genome 2007, 18:125-136.

8. Setoguchi K, Furuta M, Hirano T, Nagao T, Watanabe T, Sugimoto $Y$, Takasuga A: Cross-breed comparisons identified a critical 591-kb region for bovine carcass weight QTL (CW-2) on chromosome 6 and the lle442-Met substitution in NCAPG as a positional candidate. BMC Genet 2009, 10:43

9. Snelling WM, Allan MF, Keele JW, Kuehn LA, Thallman RM, Bennett GL, Ferrell CL, Jenkins TG, Freetly HC, Nielsen MK, Rolfe KM: Partial-genome evaluation of postweaning feed intake and efficiency of crossbred beef cattle. J Anim Sci 2011, 89:1731-1741.

10. Malik S, Roeder RG: The metazoan Mediator co-activator complex as an integrative hub for transcriptional regulation. Nat Rev Genet 2010, 11:761-772.

11. Eberlein A, Takasuga A, Setoguchi K, Pfuhl R, Flisikowski K, Fries R, Klopp N, Furbass R, Weikard R, Kuhn C: Genetic factors modulating fetal growth in cattle indicates a potential role of the non-SMC condensin I complex subunit G (NCAPG) gene. Genetics 2009, 183:951-964.

12. Seipold S, Priller FC, Goldsmith P, Harris WA, Baier H, Abdelilah-Seyfried S: Non-SMC condensing I complex proteins control chromosomal segregation and survival of proliferating cells in the zebrafish neural retina. BMC Dev Biol 2009, 9:40.

13. Soranzo N, Rivadeneira F, Chinappen-Horsley U, Malkina I, Richards JB, Hammond N, Stolk L, Nica A, Inouye M, Hofman A, Stephens J, Wheeler E, Arp P, Gwilliam R, Jhamai PM, Potter S, Chaney A, Ghori MJ, Ravindrarajah R, Ermakov S, Estrada K, Pols HA, Williams FM, McArdle WL, van Meurs JB, Loos RJ, Dermitzakis ET, Ahmadi KR, Hart DJ, Ouwehand WH, Wareham NJ, Barroso I, Sandhu MS, Strachan DP, Livshits G, Spector TD, Uitterlinden AG Deloukas P: Meta-analysis of genome-wide scans for human adult stature identifies novel Loci and associations with measures of skeletal frame size. PLoS Genet 2009, 5:e1000445.

14. Sovio U, Bennett AJ, Millwood IY, Molitor J, O'Reilly PF, Timpson NJ, Kaakinen M, Laitinen J, Haukka J, Pillas D, Tzoulaki I, Molitor J, Hoggart C, Coin LJ, Whittaker J, Pouta A, Hartikainen AL, Freimer NB, Widen E, Peltonen L, Elliott P, McCarthy MI, Jarvelin MR: Genetic determinants of height growth assessed longitudinally from infancy to adulthood in the northern Finland birth cohort 1966. PLoS Genet 2009, 5:e1000409.

15. FASS: Guide for Care and Use of Agricultural Animals in Agricultural Research and Teaching. Fed Animal Science Society. 1 edition. Savoy, IL; 1999.

16. Kuehn LA, Rohrer GA, Nonneman DJ, Thallman RM, Leymaster KA: Detection of single nucleotide polymorphisms associated with ultrasonic backfat depth in a segregating Meishan $\times$ White Composite population. J Anim Sci 2007, 85:1111-1119.

17. Rolfe KM, Snelling WM, Nielsen MK, Freetly HC, Ferrell CL, Jenkins TG: Genetic and phenotypic parameter estimates for feed intake and other traits in growing beef cattle and opportunities for selection. J Anim Sci 2011, 89:3452-3459.

18. King DA, Shackelford SD, Kuehn LA, Kemp CM, Rodriguez AB, Thallman RM, Wheeler TL: Contribution of genetic influences to animal-to-animal variation in myoglobin content and beef lean color stability. J Anim Sci 2010, 88:1160-1167.

19. Wheeler TL, Cundiff LV, Shackelford SD, Koohmaraie M: Characterization of biological types of cattle (Cycle VIII): Carcass, yield, and longissimus palatability traits. J Anim Sci 2010, 88:3070-3083.

20. Shackelford SD, Wheeler TL, Koohmaraie M: Evaluation of slice shear force as an objective method of assessing beef longissimus tenderness. J Anim Sci 1999, 77:2693-2699.

21. Rozen S, Skaletsky HJ: Primer3 on the WWW for general users and for biologist programmers. In Bioinformatics Methods and Protocols: Methods in Molecular Biology. Edited by: Krawetz S, Misener S. Totowa. New Jersey: Human Press; 2000:365-386.

22. Boldman KG, Kriese LA, Van Vleck LD, Van Tassel CP, Kachman SD: A manual for the use of MTDFREML. A set of programs to obtain estimates of variance and covariances (Draft). USDA, ARS, Lincoln, NE; 1995.

23. Kuehn LA, Keele JW, Thallman RM: Estimation of current breed differences in multibreed genetic evaluations using quantitative and molecular approaches. Beef Improvement Federation: $9^{\text {th }}$ Genetic Prediction Workshop. Prediction of Genetic Merit of Animals for Selection 2008, 49-60.

24. Goddard ME, Hayes BJ: Mapping genes for complex traits in domestic animals and their use in breeding programs. Nature Rev Genet 2009, 92:381-391.

25. Barrett JC, Fry B, Maller J, Daly MJ: Haploview: analysis and visualization of LD and haplotype maps. Bioinformatics 2005, 21:263-265.

26. Van Tassell CP, Smith TP, Matukumalli LK, Taylor JF, Schnabel RD, Lawley CT, Haudenschild CD, Moore SS, Warren WC, Sonstegard TS: SNP discovery and allele frequency estimation by deep sequencing of reduced representation libraries. Nat Methods 2008, 5:247-252.

27. Bovine HapMap Consortium: Genome-wide survey of SNP variation uncovers the genetic structure of cattle breeds. Science 2009, 324:528-532.

28. Bovine Genome Sequencing and Analysis Consortium: The genome sequence of taurine cattle: a window to ruminant biology and evolution. Science 2009, 324:522-528.

29. Weikard R, Altmaier E, Suhre K, Weinberger KM, Hammon HM, Albrecht E, Setoguchi K, Takasuga A, Kühn C: Metabolomic profiles indicate distinct physiological pathways affected by two loci with major divergent effect on Bos taurus growth and lipid deposition. Physiol Genomics 2010, 42A:79-88.

30. Yao K, Yin YL, Chu WY, Li ZQ, Deng D, Li TJ, Huan RL, Zhang JS, Tan B, Wang W, Wu G: Dietary arginine supplementation increases mTOR signaling activity in skeletal muscle of neonatal pigs. J Nutr 2008, 138:867-872.

31. Lira VA, Soltow QA, Long JHD, Betters JL, Sellman JE, Criswell DS: Nitric oxide increases GLUT4 expression and regulated AMPK signaling in skeletal muscle. Am J Physiol Endocrinol Metab 2007, 293:E1062-E10628.

32. Roy D, Perreault M, Marette A: Insulin stimulation of glucose uptake in skeletal muscles and adipose tissues in vivo is NO dependent. Am J Physiol Endocrinol Metab 1998, 274:E692-E699.

doi:10.1186/1471-2156-12-103

Cite this article as: Lindholm-Perry et al:: Association, effects and validation of polymorphisms within the NCAPG - LCORL locus located on BTA6 with feed intake, gain, meat and carcass traits in beef cattle. BMC Genetics 2011 12:103. 